Министерство образования Республики Беларусь БЕЛОРУССКИЙ НАЦИОНАЛЬНЫЙ ТЕХНИЧЕСКИЙ УНИВЕРСИТЕТ

Кафедра «Экономика и организация энергетики»

\title{
ЭКОНОМИКО-МАТЕМАТИЧЕСКИЕ МЕТОДЫ
} И МОДЕЛИ

Методические указания к контрольным работам для студентов заочной формы обучения специальности 1-270101 «Экономика и организация производства» направления 1-27 01 01-10 «Энергетика»

Минск

БН ТУ

2011 
УДК 620.9:330.4

ББК 65.631я7

Э 40

\author{
С о с т а в и т ел и: \\ B.Н. Нагорнов, А.В. Куприк \\ Р е ц е н $з$ е н т ы: \\ Т.Ф. Манцерова, В.М. Холопик
}

В методических указаниях приведены рекомендации по выполнению контрольной работы по курсу «Экономико-математические методы и модели» для студентов заочной формы обучения специальности 1-27 0101 «Экономика и организация производства» направления 1-27 01 01-10 «Энергетика».

Контрольная работа включает в себя задания, решение которых позволит закрепить теоретический материал и получить навыки соответствующих расчетов и анализа.

Разработаны также индивидуальные задания по 30 вариантам, методика выполнения которых кратко изложена в каждом разделе работы.

(C) БНТУ, 2011 


\section{1. ДЕТЕРМИНИРОВАННЫЕ ЛИНЕЙНЫЕ МОДЕЛИ}

\section{1 Постановка задачи линейного программирования}

Задача линейного программирования (ЛП) в общем виде записывается следующим образом (1.1)-(1.4). Каждой задаче ЛП может быть поставлена в соответствие другая вполне определенная задача ЛП, такая, что при решении одной из них одновременно решается и другая. Эти задачи названы парой взаимодвойственных задач. Любой задаче ЛП (1.1)-(1.4) можно поставить в соответствие двойственную задачу вида (1.5)-(1.8):

$$
\begin{aligned}
& F=\sum_{j=1}^{n} c_{j} \cdot x_{j} \rightarrow \max (\min ), \\
& \left\{\begin{array}{l}
\sum_{j=1}^{n} a_{i j} \cdot x_{j} \leq b_{i}, i=\overline{1, k}, k \leq m, \\
\sum_{j=1}^{n} a_{i j} \cdot x_{j}=b_{i}, i=\overline{k+1, m}, \\
x_{j} \geq 0, j=\overline{1, l}, \mid \leq n ;
\end{array}\right. \\
& f=\sum_{i=1}^{n} b_{i} \cdot y_{i} \rightarrow \min (\max ), \\
& \left\{\begin{array}{l}
\sum_{i=1}^{m} a_{i j} \cdot y_{i} \geq c_{j}, j=\overline{1, k}, \\
\sum_{i=1}^{m} a_{i j} \cdot y_{i}=c_{j}, j=\overline{k+1, n,} \\
y_{i} \geq 0, i=\overline{1, s} .
\end{array}\right.
\end{aligned}
$$


где $x_{j}$ - неизвестные величины;

$a_{i j}, b_{i}, c_{j}$ - заданные действительные числа;

(1.1), (1.5) - целевые функции (ЦФ);

(1.2), (1.3), (1.6), (1.7) - основные ограничения задачи;

(1.4) - не основные ограничения;

уі имеет произвольный знак для $\mathrm{i}=\overline{\mathrm{s}+1, \mathrm{~m}}$.

Для решения задач ЛП может быть использован графический метод, симплекс-метод, метод искусственного базиса, модифицированный симплекс-метод и двойственный симплекс-метод.

\section{Правила составления двойственных задач:}

1) число неизвестных одной задачи равно числу ограничений второй;

2) матрицы коэффициентов системы ограничений получаются одна из другой путем транспонирования;

3) знаки неравенств в системе ограничений заменяют на противоположные, например, $\leq$ на $\geq$, и наоборот;

4) свободные члены ограничений исходной задачи становятся коэффициентами целевой функции двойственной задачи и, наоборот, коэффициенты целевой функции исходной задачи преобразуются в свободные члены ограничений двойственной;

5) критерий оптимальности целевой функции заменяется на противоположный, например, max на min.

\section{2. Анализ линейных моделей на чувствительность (устойчивость)}

Анализ линейных моделей на чувствительность - это процесс, реализуемый после нахождения оптимального решения. При таком анализе рассматривается комплекс линейных оптимизационных моделей. Это придает задаче определенную динамичность, что позволяет проанализировать влияние возможных изменений исходных данных на полученное ранее оптимальное решение. 
Неизбежное колебание значений таких экономических параметров, как цены на продукцию и сырье, запасы сырья, спрос на рынке и т. д., может привести к неоптимальности или непригодности прежнего режима работы. Для учета подобных ситуаций проводится анализ чувствительности, т. е. анализ того, как возможные изменения параметров исходной модели повлияют на полученное ранее оптимальное решение задачи ЛП.

Отсутствие такого анализа может привести к тому, что полученное оптимальное решение устареет еще до своей реализации.

\section{Основные задачи анализа на чувствительность:}

1. Анализ изменения запасов ресурсов позволяет ответить на вопросы:

- на сколько можно увеличить запас некоторого дефицитного ресурса с целью улучшения значения целевой функции;

- на сколько можно уменьшить запас некоторого недефицитного ресурса с сохранением полученного ранее оптимального значения целевой функции.

Если ресурс израсходован полностью, его относят к разряду дефицитных. Ресурс в избытке называют недефицитным. Объем недефицитного ресурса можно уменьшить на величину избытка без изменения значения целевой функции. Объем дефицитного ресурса не следует увеличивать сверх того предела, когда соответствующее ему ограничение становится избыточным.

2. Определение наиболее выгодного ресурса позволяет ответить на вопрос, какому из дефицитных ресурсов следует отдать предпочтение при вложении дополнительных средств. Вводится характеристика ценности единицы ресурса:

$$
\mathrm{y}_{\mathrm{i}}=\frac{\text { максимальное прирамение иелевой функиии }}{\text { максимальный допустимый прирост і-го ресурса }} \text {, (1.9) }
$$

где $\mathrm{y}_{\mathrm{i}}$ - теневая цена ресурса.

Теневая цена показывает, на сколько изменится значение целевой функции при изменении запаса ресурса на единицу. 
Теневая цена позволяет определить статус ресурса. У недефицитного ресурса теневая цена равна нулю, положительное значение теневой цены говорит о дефицитности данного ресурса.

Значение теневой цены ресурсов - это решение задачи, двойственной к данной.

3. Определение пределов изменения коэффициентов ЦФ позволяет ответить на вопросы:

- каков диапазон изменения того или иного коэффициента ЦФ, при котором не происходит изменение оптимального решения;

- на сколько следует изменить тот или иной коэффициент ЦФ, чтобы сделать дефицитный ресурс недефицитным, и наоборот.

\section{3. Экономическая интерпретация решения задач ЛП}

ПРИМЕР 1.1 Предприятие изготавливает два вида продукции $-\Pi_{1}$ и П2. Для производства продукции используется два вида сырья - А и Б. Максимально возможные запасы сырья, расход сырья на единицу приведены в таблице.

\begin{tabular}{|c|c|c|c|}
\hline \multirow{2}{*}{ Сырье } & \multicolumn{2}{|c|}{ Расход сырья на единицу } & \multirow{2}{*}{ Запас } \\
\cline { 2 - 3 } & $\Pi_{1}$ & $\Pi_{2}$ & 9 \\
\hline А & 2 & 3 & 13 \\
\hline Б & 3 & 2 & 9 \\
\hline
\end{tabular}

Опыт работы показал, что суточный спрос на продукцию $\Pi_{1}$ никогда не превышает спрос на продукцию П 2 больше, чем на единицу. Спрос на продукцию П2 не превышает 2-х единиц в сутки. Цена реализации П1 составляет 3 денежные единицы, $\Pi_{2}-4$ денежные единицы.

Какой объем продукции каждого вида должно выпускать предприятие, чтобы максимизировать свой суточный доход?

РЕШЕНИЕ. Введем переменные: $x_{1}$ - объем выпуска продукции П 1 ; 2 - объем выпуска продукции П2. Математическая модель задачи и задача в каноническом виде имеют следующий вид: 


$$
\begin{aligned}
& \mathrm{F}=3 \cdot \mathrm{x}_{1}+4 \cdot \mathrm{x}_{2} \rightarrow \max \\
& \left\{\begin{array}{l}
2 \cdot \mathrm{x}_{1}+3 \cdot \mathrm{x}_{2} \leq 9 ; \\
3 \cdot \mathrm{x}_{1}+2 \cdot \mathrm{x}_{2} \leq 13 ; \\
\mathrm{x}_{1}-\mathrm{x}_{2} \leq 1 ; \\
x_{2} \leq 2 ; \\
x_{1} \geq 0 ; x_{2} \geq 0
\end{array}\right. \\
& \mathrm{F}=3 \cdot \mathrm{x}_{1}+4 \cdot \mathrm{x}_{2}+0 \cdot \mathrm{x}_{3}+0 \cdot \mathrm{x}_{4}+0 \cdot \mathrm{x}_{5}+0 \cdot \mathrm{x}_{6} \rightarrow \max \\
& \left\{\begin{array}{l}
2 \cdot \mathrm{x}_{1}+3 \cdot \mathrm{x}_{2}+x_{3}=9 ; \\
3 \cdot \mathrm{x}_{1}+2 \cdot \mathrm{x}_{2}+x_{4}=13 ; \\
\mathrm{x}_{1}-\mathrm{x}_{2}+x_{5}=1 ; \\
x_{2}+x_{6}=2 ; \\
x_{\mathrm{j}} \geq 0 ; \mathrm{j}=\overline{1,6}
\end{array}\right.
\end{aligned}
$$

Дополнительные переменные $\left(x_{3}, x_{4}, x_{5}, x_{6}\right.$, которые прибавили к левым частям соответствующих неравенств) имеют экономический смысл: они показывают величину неиспользованного ресурса. Так, в данном примере $x_{3}$ показывает величину неиспользованного сырья А, $x_{4}$ - сырья Б, $x_{5}$ показывает неиспользованную разницу в спросе на продукцию П и и $\Pi_{2}$, $x_{6}$ - невостребованное количество продукции П2.

Составим исходную симплекс-таблицу:

\begin{tabular}{|c|c|c|c|c|c|c|c|}
\hline $\begin{array}{c}\text { Базисные } \\
\text { переменные }\end{array}$ & Решение & $x_{1}$ & $x_{2}$ & $x_{3}$ & $x_{4}$ & $x_{5}$ & $x_{6}$ \\
\hline$x_{3}$ & 9 & 2 & 3 & 1 & 0 & 0 & 0 \\
\hline$x_{4}$ & 13 & 3 & 2 & 0 & 1 & 0 & 0 \\
\hline$x_{5}$ & 1 & 1 & -1 & 0 & 0 & 1 & 0 \\
\hline$x_{6}$ & 2 & 0 & 1 & 0 & 0 & 0 & 1 \\
\hline$-\mathrm{F}$ & 0 & -3 & -4 & 0 & 0 & 0 & 0 \\
\hline
\end{tabular}


Решая задачу симплекс-методом, получим следующую итоговую симплекс-таблицу:

\begin{tabular}{|c|c|c|c|c|c|c|c|}
\hline $\begin{array}{c}\text { Базисные } \\
\text { переменные }\end{array}$ & Решение & $x_{1}$ & $x_{2}$ & $x_{3}$ & $x_{4}$ & $x_{5}$ & $x_{6}$ \\
\hline$x_{1}$ & 2,4 & 1 & 0 & 0,2 & 0 & 0,6 & 0 \\
\hline$x_{4}$ & 3 & 0 & 0 & -1 & 1 & -1 & 0 \\
\hline$x_{6}$ & 0,6 & 0 & 0 & $-0,2$ & 0 & 0,4 & 1 \\
\hline$x_{2}$ & 1,4 & 0 & 1 & 0,2 & 0 & $-0,4$ & 0 \\
\hline $\mathrm{F}$ & 12,8 & 0 & 0 & 1,4 & 0 & 0,2 & 0 \\
\hline
\end{tabular}

Итоговая симплекс-таблица позволяет ответить на ряд вопросов, касающихся анализа на чувствительность:

1. Остаточные (балансовые или дополнительные) переменные $x_{3}, x_{4}, x_{5}, x_{6}$ позволяют определить статус ресурсов. Если значение остаточной переменной равно нулю ( $x_{3}$ и $\left.x_{5}\right)$, то ресурс израсходован полностью, т. е. является дефицитным.

Положительное значение остаточной переменной $\left(x_{4}=3\right.$, $\left.x_{6}=0,6\right)$ говорит о недефицитности соответствующего ресурса. Это и есть величина избытка. Соответствующие ресурсы можно уменьшить на полученную величину без изменения значения ЦФ.

2. Теневая цена ресурсов указана в последней строке симплекс-таблицы. Наиболее выгодный - первый ресурс (сырье A, ему соответствует переменная $\left.x_{3}\right)$, т. к. он имеет наибольшую теневую цену $y_{1}=1,4$. Поэтому дополнительные капиталовложения в первую очередь следует направлять на увеличение запаса сырья А (первый ресурс) и лишь затем - на формирование разницы в спросе на продукцию П1 и П2.

3. Максимальное изменение запаса ресурса.

Пусть запас первого ресурса (сырье А) изменится на величину $\Delta_{1}$, тогда результирующая симплекс-таблица примет следующий вид: 


\begin{tabular}{|c|c|c|c|c|c|c|c|}
\hline $\begin{array}{c}\text { Базисные } \\
\text { переменные }\end{array}$ & Решение & $x_{1}$ & $x_{2}$ & $x_{3}$ & $x_{4}$ & $x_{5}$ & $x_{6}$ \\
\hline$x_{1}$ & $2,4+0,2 \cdot \Delta_{1}$ & 1 & 0 & 0,2 & 0 & 0,6 & 0 \\
\hline$x_{4}$ & $3-1 \cdot \Delta_{1}$ & 0 & 0 & -1 & 1 & -1 & 0 \\
\hline$x_{6}$ & $0,6-0,2 \cdot \Delta_{1}$ & 0 & 0 & $-0,2$ & 0 & 0,4 & 1 \\
\hline$x_{2}$ & $1,4+0,2 \cdot \Delta_{1}$ & 0 & 1 & 0,2 & 0 & $-0,4$ & 0 \\
\hline $\mathrm{F}$ & $12,8+1,4 \cdot \Delta_{1}$ & 0 & 0 & 1,4 & 0 & 0,2 & 0 \\
\hline
\end{tabular}

Так как изменение величины ресурса сказывается только на элементах столбца «Решение», то это может повлиять только на допустимость решения, поэтому должна выполняться система

$$
\left\{\begin{array}{l}
2,4+0,2 \cdot \Delta_{1} \geq 0 \\
3-1 \cdot \Delta_{1} \geq 0 \\
0,6-0,2 \cdot \Delta_{1} \geq 0 \\
1,4+0,2 \cdot \Delta_{1} \geq 0
\end{array}\right.
$$

Решая систему неравенств, получим: $-7 \leq \Delta_{1} \leq 3$. Таким образом, уменьшение запаса сырья А (первый ресурс) более чем на 7 единиц или увеличение более чем на 3 единицы приведет к недопустимости полученного решения и новой совокупности базисных переменных. Внутри указанного интервала решение будет действительным. Запас сырья А должен быть в следующих пределах:

$$
\begin{gathered}
9-7 \leq 9+\Delta_{1} \leq 9+3 \\
2 \leq \text { запас сырья } \mathrm{A} \leq 12 .
\end{gathered}
$$

Вывод: запас сырья А можно увеличить на 3 единицы с 9 до 12, это приведет к увеличению ЦФ с 12,8 до 17 единиц $(12,8+1,4 \cdot 3=17)$.

4. Анализ на чувствительность оптимального решения к изменению коэффициентов ЦФ. 
Пусть доход, получаемый с единицы продукции П1, изменится на величину $\Delta_{1}$, тогда итоговая симплекс-таблица примет следующий вид:

\begin{tabular}{|c|c|c|c|c|c|c|c|}
\hline $\begin{array}{c}\text { Базисные } \\
\text { переменные }\end{array}$ & Решение & $x_{1}$ & $x_{2}$ & $x_{3}$ & $x_{4}$ & $x_{5}$ & $x_{6}$ \\
\hline$x_{1}$ & 2,4 & 1 & 0 & 0,2 & 0 & 0,6 & 0 \\
\hline$x_{4}$ & 3 & 0 & 0 & -1 & 1 & -1 & 0 \\
\hline$x_{6}$ & 0,6 & 0 & 0 & $-0,2$ & 0 & 0,4 & 1 \\
\hline$x_{2}$ & 1,4 & 0 & 1 & 0,2 & 0 & $-0,4$ & 0 \\
\hline $\mathrm{F}$ & $12,8+2,4 \cdot \Delta_{1}$ & 0 & 0 & $1,4+0,2 \cdot \Delta_{1}$ & 0 & $0,2+0,6 \cdot \Delta_{1}$ & 0 \\
\hline
\end{tabular}

$x_{3}$ и $x_{5}$ не вошли в базис, должно выполняться:

$$
\left\{\begin{array}{l}
1,4+0,2 \cdot \Delta_{1} \geq 0, \\
0,2+0,6 \cdot \Delta_{1} \geq 0 .
\end{array} \quad-1 / 3 \leq \Delta_{1} \leq+\infty .\right.
$$

При изменении цены на первый вид продукции от $8 / 3$ до $+\infty$ оптимальные значения переменных останутся неизменными:

$$
3-1 / 3 \leq Ц_{1}=3 \leq 3+\infty=\infty .
$$

\section{4. Экономическая интерпретация двойственности}

Задача, двойственная к задаче об ассортименте продукции, рассмотренной в примере 1.1, имеет вид:

$$
\begin{aligned}
& F=9 \cdot y_{1}+13 \cdot y_{2}+y_{3}+2 \cdot y_{4} \rightarrow \min , \\
& \left\{\begin{array}{l}
2 \cdot y_{1}+3 \cdot y_{2}+y_{3} \geq 3, \\
3 \cdot y_{1}+2 \cdot y_{2}-y_{3}+y_{4} \geq 4, \\
y_{i} \geq 0 ; i=\overline{1,4} .
\end{array}\right.
\end{aligned}
$$


Аналогично как и для прямой задачи, двойственную задачу представляют в каноническом виде, т. е. вводят дополнительные переменные $y_{5}, y 6$, которые прибавляют к левым частям соответствующих неравенств. В целевую функцию все дополнительные переменные вводят с коэффициентами, равными нулю.

Решая двойственную задачу симплекс-методом, получим следующую итоговую таблицу:

\begin{tabular}{|c|c|c|c|c|c|c|c|}
\hline $\begin{array}{c}\text { Базисные } \\
\text { переменные }\end{array}$ & Решение & $\mathrm{y}_{1}$ & $\mathrm{y}_{2}$ & $\mathrm{y}_{3}$ & $\mathrm{y}_{4}$ & $\mathrm{y}_{5}$ & $\mathrm{y}_{6}$ \\
\hline $\mathrm{y}_{1}$ & 1,4 & 1 & 1 & 0 & 0,2 & $-0,2$ & $-0,2$ \\
\hline $\mathrm{y}_{3}$ & 0,2 & 0 & 1 & 1 & $-0,4$ & $-0,6$ & 0,4 \\
\hline $\mathrm{f}$ & 12,8 & 0 & -3 & 0 & $-0,6$ & $-2,4$ & $-1,4$ \\
\hline
\end{tabular}

Переменные уі двойственной задачи называют двойственными оценками, они представляют собой теневые цены соответствующих ресурсов прямой задачи.

Анализ на чувствительность оптимального решения базируется на следующих свойствах двойственных оценок (ДО):

1. ДО характеризуют дефицитность ресурсов: чем больше значение ДО, тем более дефицитным является ресурс. Для недефицитных ресурсов $\mathrm{y}_{\mathrm{i}}=0$.

2. ДО показывают, как влияют изменения в правой части ограничений (запасов ресурсов) на значение ЦФ.

Практический интерес представляет верхняя и нижняя границы изменения ресурсов, в которых значения оценок остаются неизменными.

Для дефицитных ресурсов:

нижняя граница $\Delta b_{i}^{\left({ }^{*}\right)}=\min _{k}\left\{\frac{x_{k}}{d_{k i}}\right\}$ для $d_{k i}>0$;

верхняя граница $\Delta b_{i}^{(в)}=\max _{k}\left\{\left|\frac{x_{k}}{d_{k i}}\right|\right\}$ для $d_{k i}<0$, 
где і - номер ресурса;

k - индекс базисной переменной;

$x_{k}$ - оптимальное значение базисной переменной;

$d_{\text {ki }}$ - элементы матрицы коэффициентов при базисных переменных в итоговой симплекс-таблице прямой задачи.

Для примера 1.1 для дефицитных ресурсов (см. итоговую табл. пункта 1.3):

$$
\begin{gathered}
\Delta b_{1}^{(\text {н) }}=\min _{k}\left\{\frac{x_{1}}{d_{11}} ; \frac{x_{2}}{d_{21}}\right\}=\min _{k}\left\{\frac{2,4}{0,2} ; \frac{1,4}{0,2}\right\}=7 \\
\Delta b_{1}^{(\text {в) }}=\max _{k}\left\{\left|\frac{x_{4}}{d_{41}}\right| ;\left|\frac{x_{6}}{d_{61}}\right|\right\}=\max _{k}\left\{\left|\frac{3}{-1}\right| ;\left|\frac{0,6}{-0,2}\right|\right\}=3 ; \\
\Delta b_{3}^{(\text {н) }}=\min _{k}\left\{\frac{x_{1}}{d_{13}} ; \frac{x_{6}}{d_{63}}\right\}=\min _{k}\left\{\frac{2,4}{0,6} ; \frac{0,6}{0,4}\right\}=1,5 ; \\
\Delta b_{3}^{(\text {в) }}=\max _{k}\left\{\left|\frac{x_{4}}{d_{43}}\right| ;\left|\frac{x_{2}}{d_{23}}\right|\right\}=\max _{k}\left\{\left|\frac{3}{-1}\right| ;\left|\frac{1,4}{-0,4}\right|\right\}=3,5 .
\end{gathered}
$$

Для недефицитных ресурсов верхняя граница интервала устойчивости определяется исходными данными, а нижняя равна величине фактически израсходованных ресурсов.

Таким образом, интервалы устойчивости оценок по отношению к изменению ресурсов будут равны:
1) $[9-7 ; 9+3]=[2 ; 12]$;
2) $[13-3 ; 13]=[10 ; 13]$;
3) $[1-1,5 ; 1+3,5]=[-0,5 ; 4,5]$;
4) $[2-0,6 ; 2]=[1,4 ; 2]$.

Если изменения запасов ресурсов находятся в пределах устойчивости двойственных оценок, их раздельное влияние на 
значение ЦФ равно произведению двойственной оценки и величины изменения запасов ресурса.

$$
\begin{array}{ll}
\Delta \mathrm{F}_{1}=1,4 \cdot(12-2)=14 ; & \Delta \mathrm{F}_{2}=0 \cdot(13-10)=0 ; \\
\Delta \mathrm{F}_{3}=0,2 \cdot(4,5-(-0,5))=1 ; & \Delta \mathrm{F}_{4}=0 \cdot(2-1,4)=0 .
\end{array}
$$

Суммарное возможное увеличение ЦФ составит:

$$
\Delta \mathrm{F}=1,4 \cdot 3+0,2 \cdot 3,5=4,9 .
$$

3. ДО являются показателем эффективности производства отдельных видов продукции с точки зрения критерия оптимальности. С этой позиции в оптимальный план может быть включена лишь та продукция, для которой выполняется условие

$$
\sum_{i=1}^{m} a_{i j} \cdot y_{i} \leq c_{j} .
$$

Например, введение в план третьего вида продукции с технологическими коэффициентами $a_{13}=3 ; a_{2} 3=1$ и ценой $c 3=8$ выгодно, поскольку выполняется условие: $3 \cdot 1,4+1 \cdot 0=4,2 \leq 8$. Математическая модель задачи примет следующий вид:

$$
\begin{aligned}
& \mathrm{F}=3 \cdot \mathrm{x}_{1}+4 \cdot \mathrm{x}_{2}+8 \cdot x_{3} \rightarrow \max \\
& \left\{\begin{array}{l}
2 \cdot \mathrm{x}_{1}+3 \cdot \mathrm{x}_{2}+3 \cdot x_{3} \leq 9 \\
3 \cdot \mathrm{x}_{1}+2 \cdot \mathrm{x}_{2}+x_{3} \leq 13 \\
\mathrm{x}_{1}-\mathrm{x}_{2} \leq 1 \\
x_{2} \leq 2 \\
x_{1} \geq 0 ; x_{2} \geq 0, x_{3} \geq 0
\end{array}\right.
\end{aligned}
$$

4. ДО позволяют производить сравнение суммарных условных затрат и результатов. Так, например, приобретение двух единиц первого ресурса (сырье А) по цене $c=0,5$ денежных единиц целесообразно, т. к.: 
a) изменение ресурса находится в пределах устойчивости ДО;

б) $\Delta$ Доход $=\Delta F=2 \cdot 1,4=2,8$;

$\triangle$ Расход $=2,0 \cdot 0,5=1$;

$\Delta$ Прибыль $=\Delta$ Доход $-\Delta$ Расход $=2,8-1=1,8>0$, т. е. прибыль увеличивается.

\section{Задание 1}

На предприятии выпускают $n$ видов продукции $\Pi_{j}(j=\overline{1, n})$. При ее изготовлении используются ресурсы $P_{1}, P_{2}$ и $P_{3}$. Размеры допустимых затрат ресурсов ограничены соответственно величинами $b_{1}, b_{2}$ и $b_{3}$. Расход ресурса $i$-го вида $(i=1,2,3)$ на единицу продукции ј-го вида составляет $a_{i j}$ денежных единиц. Цена единицы продукции ј-го вида равна с денежных единиц.

Задание, соответствующее номеру варианта (по порядковому номеру в списке группь), приведено в таблицах 1.1, 1.2

Требуется:

1) составить математическую модель задачи, позволяющую найти сбалансированный план выпуска продукции, обеспечивающий предприятию максимальный доход;

2) найти оптимальный план выпуска продукции каждого вида симплекс-методом (дать содержательный ответ, раскрыв экономический смысл всех переменных, приведенных в решении задачи);

3) составить математическую модель двойственной задачи и определить ее решение по итоговой симплекс-таблице исходной задачи;

4) определить статус ресурсов и теневую цену всех ресурсов, найти наиболее выгодный ресурс;

5) определить максимальное изменение запаса каждого реcypca;

6) определить пределы изменения каждого коэффициента целевой функции;

7) с помощью двойственных оценок определить верхнюю и нижнюю границы изменения запаса каждого ресурса (найти 14 
интервалы устойчивости оценок по отношению к изменению ресурсов);

8) определить, выгодно ли введение в план производства нового вида продукции с технологическими коэффициентами $a_{\text {in }}$ и ценой $c$, представленными в таблице 1.2 .

Таблица 1.1

\begin{tabular}{|c|c|c|c|c|c|c|c|c|c|c|c|c|c|c|c|}
\hline \multirow{2}{*}{$\begin{array}{l}\text { Па- } \\
\text { ра- }\end{array}$} & \multicolumn{15}{|c|}{ Номер варианта } \\
\hline & 1 & 2 & 3 & 4 & 5 & 6 & 7 & 8 & 9 & 10 & 11 & 12 & 13 & 14 & 15 \\
\hline$n$ & 4 & 3 & 4 & 3 & 3 & 3 & 3 & 4 & 3 & 3 & 3 & 3 & 4 & 3 & 4 \\
\hline$b_{1}$ & 20 & 150 & 280 & 1200 & 600 & 24 & 500 & 100 & 360 & 180 & 25 & 200 & 220 & 1200 & 600 \\
\hline$b_{2}$ & 37 & 180 & 80 & 150 & 30 & 10 & 550 & 260 & 192 & 210 & 47 & 180 & 80 & 150 & 30 \\
\hline$b_{3}$ & 30 & 120 & 250 & 3000 & 144 & 6 & 200 & 370 & 180 & 244 & 30 & 150 & 250 & 3000 & 144 \\
\hline$a_{11}$ & 2 & 2 & 2 & 15 & 10 & 5 & 2 & 2,5 & 18 & 4 & 2 & 2 & 2 & 15 & 10 \\
\hline$a_{12}$ & 2 & 3 & 1 & 20 & 20 & 7 & 1 & 2,5 & 15 & 2 & 2 & 6 & 1 & 20 & 20 \\
\hline$a_{13}$ & 3 & 4 & 1 & 25 & 23 & 4 & 0 & 2 & 12 & 1 & 3 & 4 & 1 & 25 & 20 \\
\hline$a_{14}$ & 0 & - & 1 & - & - & - & - & 1,5 & - & - & - & - & 1 & - & \begin{tabular}{|l|}
15 \\
\end{tabular} \\
\hline$a_{21}$ & 3 & 11 & 1 & 2 & 1 & 5 & 0 & 4 & 6 & 3 & 3 & \begin{tabular}{|l|}
11 \\
\end{tabular} & 1 & 2 & 1 \\
\hline$a_{22}$ & 1 & 4 & 0 & 3 & 1 & 2 & 2 & 10 & 4 & 1 & 1 & 7 & 0 & 3 & 5 \\
\hline$a_{23}$ & 1 & 5 & 1 & 2,5 & 1 & 1 & 1 & 4 & 8 & 3 & 1 & 5 & 1 & 2,5 & 1 \\
\hline$a_{24}$ & 2 & - & 1 & - & - & - & - & 6 & - & - & - & - & 3 & - & 7 \\
\hline$a_{31}$ & 0 & 3 & 1 & 35 & 5 & 2 & 0 & 8 & 5 & 1 & 0 & 3 & 1 & 35 & 5 \\
\hline$a_{32}$ & 1 & 4 & 2 & 60 & 6 & 1 & 1 & 7 & 3 & 2 & 1 & 4 & 2 & 60 & 8 \\
\hline a33 & 1 & 2 & 1 & 60 & 6 & 1 & 0 & 4 & 3 & 5 & 1 & 0 & 1 & 60 & 6 \\
\hline$a_{34}$ & 4 & - & 0 & - & - & - & - & 10 & - & - & - & - & 0 & - & 4 \\
\hline$C_{1}$ & 11 & 8 & 4 & 300 & 35 & 18 & 3 & 40 & 9 & 10 & 11 & 5 & 4 & 300 & \begin{tabular}{|l|}
35 \\
\end{tabular} \\
\hline $\mathrm{C}_{2}$ & 6 & 7 & 3 & 250 & 60 & 12 & 4 & 50 & 10 & 14 & 6 & 7 & 3 & 250 & 60 \\
\hline$C_{3}$ & 9 & 6 & 6 & 450 & 63 & 8 & 1 & 100 & 16 & 12 & 10 & 6 & 7 & 450 & \begin{tabular}{|l|}
63 \\
\end{tabular} \\
\hline $\mathrm{C}_{4}$ & 6 & - & 7 & - & - & - & - & 80 & - & - & - & - & 7 & - & 40 \\
\hline \multirow{2}{*}{$\begin{array}{l}\text { Пара- } \\
\text { метр }\end{array}$} & \multicolumn{15}{|c|}{ Номер варианта } \\
\hline & 16 & 17 & 18 & 19 & 20 & 21 & 22 & 23 & 24 & 25 & 26 & 27 & 28 & 29 & 30 \\
\hline $\bar{n}$ & 3 & 3 & 4 & 4 & 3 & 4 & 4 & 4 & 3 & 3 & 3 & 4 & 3 & 4 & 3 \\
\hline $\mathrm{b}_{1}$ & 24 & 500 & 100 & 300 & 180 & 20 & 250 & 128 & 1200 & 1020 & 80 & 10 & 60 & 80 & 90 \\
\hline $\mathrm{b}_{2}$ & 14 & 550 & 260 & 200 & 140 & 37 & 280 & 150 & 150 & 30 & 30 & 50 & \begin{tabular}{|l|}
80 \\
\end{tabular} & 40 & 60 \\
\hline$b_{3}$ & 6 & 200 & 370 & 180 & 244 & 30 & 220 & 250 & 3000 & \begin{tabular}{|l|}
140 \\
\end{tabular} & 50 & 40 & 50 & 55 & 70 \\
\hline$a_{11}$ & 5 & 2 & 2,5 & 18 & 4 & 2 & 4 & 2 & 15 & 15 & 5 & 4 & 3 & 7 & 8 \\
\hline$a_{12}$ & 7 & 1 & 2,5 & 14 & 2 & 2 & 5 & 1 & 20 & 20 & 8 & 9 & 5 & 4 & 7 \\
\hline
\end{tabular}


Окончание табл. 1.1

\begin{tabular}{|c|c|c|c|c|c|c|c|c|c|c|c|c|c|c|c|}
\hline \multirow{2}{*}{$\begin{array}{c}\text { Пара- } \\
\text { метр }\end{array}$} & \multicolumn{110}{|c|}{ Номер варианта } \\
\cline { 2 - 18 } & 16 & 17 & 18 & 19 & 20 & 21 & 22 & 23 & 24 & 25 & 26 & 27 & 28 & 29 & 30 \\
\hline $\mathrm{a}_{13}$ & 4 & 0 & 2 & 12 & 5 & 3 & 6 & 1 & 25 & 23 & 20 & 15 & 12 & 13 & 10 \\
\hline $\mathrm{a}_{14}$ & - & - & 1,5 & - & - & 0 & - & 1 & - & - & - & 5 & - & 6 & - \\
\hline $\mathrm{a}_{21}$ & 5 & 0 & 4 & 6 & 3 & 3 & 11 & 1 & 2 & 10 & 8 & 8 & 7 & 5 & 9 \\
\hline $\mathrm{a}_{22}$ & 2 & 2 & 10 & 4 & 1 & 1 & 4 & 0 & 3 & 12 & 7 & 10 & 12 & 10 & 11 \\
\hline $\mathrm{a}_{23}$ & 7 & 1 & 4 & 8 & 3 & 1 & 5 & 1 & 2,5 & 15 & 8 & 10 & 12 & 14 & 15 \\
\hline $\mathrm{a}_{24}$ & - & - & 6 & - & - & 2 & - & 3 & - & - & - & 5 & - & 6 & - \\
\hline $\mathrm{a}_{31}$ & 2 & 0 & 8 & 5 & 1 & 0 & 3 & 1 & 35 & 5 & 5 & 4 & 8 & 9 & 10 \\
\hline $\mathrm{a}_{32}$ & 1 & 1 & 7 & 3 & 2 & 1 & 4 & 2 & 60 & 6 & 6 & 12 & 13 & 14 & 15 \\
\hline $\mathrm{a}_{33}$ & 1 & 0 & 4 & 3 & 5 & 1 & 6 & 1 & 60 & 6 & 6 & 5 & 7 & 0 & 1 \\
\hline $\mathrm{a}_{34}$ & - & - & 10 & - & - & 4 & - & 0 & - & - & - & 6 & - & 8 & - \\
\hline $\mathrm{C}_{1}$ & 18 & 3 & 40 & 9 & 16 & 11 & 8 & 4 & 300 & 35 & 8 & 5 & 1 & 0 & 3 \\
\hline $\mathrm{C}_{2}$ & 10 & 4 & 50 & 12 & 14 & 25 & 7 & 3 & 250 & 60 & 7 & 3 & 2 & 1 & 4 \\
\hline $\mathrm{C}_{3}$ & 8 & 1 & 100 & 16 & 12 & 15 & 6 & 6 & 450 & 45 & 4 & 3 & 5 & 1 & 6 \\
\hline $\mathrm{C}_{4}$ & - & - & 80 & - & - & 40 & - & 7 & - & - & - & 10 & - & 9 & - \\
\hline
\end{tabular}

Таблица 1.2

\begin{tabular}{|c|c|c|c|c|}
\hline \multirow{2}{*}{$\begin{array}{c}\text { Номер } \\
\text { варианта }\end{array}$} & \multicolumn{4}{|c|}{ Параметр } \\
\hline & $a_{1 n}$ & $a_{2 n}$ & $a_{3 n}$ & $c$ \\
\hline 1 & 3 & 5 & 6 & 10 \\
\hline 2 & 5 & 5 & 0 & 5 \\
\hline 3 & 10 & 2 & 10 & 6 \\
\hline 4 & 14 & 9 & 15 & 180 \\
\hline 5 & 12 & 7 & 12 & 40 \\
\hline 6 & 13 & 8 & 4 & 10 \\
\hline 7 & 15 & 2 & 8 & 5 \\
\hline 8 & 10 & 4 & 9 & 75 \\
\hline 9 & 9 & 8 & 6 & 12 \\
\hline 10 & 8 & 12 & 7 & 10 \\
\hline 11 & 7 & 3 & 12 & 9 \\
\hline 12 & 5 & 10 & 13 & 3 \\
\hline 13 & 10 & 4 & 15 & 5 \\
\hline 14 & 11 & 15 & 18 & 200 \\
\hline 15 & 12 & 10 & 14 & 56 \\
\hline 16 & 14 & 11 & 16 & 12 \\
\hline 17 & 15 & 12 & 21 & 6 \\
\hline
\end{tabular}


Окончание табл. 1.2

\begin{tabular}{|c|c|c|c|c|}
\hline \multirow{2}{*}{$\begin{array}{c}\text { Номер } \\
\text { варианта }\end{array}$} & \multicolumn{4}{|c|}{ Параметр } \\
\hline & $a_{1 n}$ & $a_{2 n}$ & $a_{3 n}$ & $c$ \\
\hline 18 & 13 & 16 & 25 & 15 \\
\hline 19 & 16 & 21 & 14 & 15 \\
\hline 20 & 14 & 14 & 14 & 12 \\
\hline 21 & 20 & 17 & 9 & 30 \\
\hline 22 & 21 & 8 & 5 & 8 \\
\hline 23 & 5 & 9 & 6 & 3 \\
\hline 24 & 0 & 7 & 8 & 300 \\
\hline 25 & 8 & 6 & 7 & 40 \\
\hline 26 & 4 & 6 & 9 & 50 \\
\hline 27 & 7 & 6 & 5 & 9 \\
\hline 28 & 9 & 3 & 6 & 10 \\
\hline 29 & 6 & 0 & 7 & 15 \\
\hline 30 & 6 & 8 & 9 & 12 \\
\hline
\end{tabular}

\section{2 ТРАНСПОРТНЫЕ МОДЕЛИ}

\section{1 Транспортная задача и ее особенности}

При планировании перевозок однородных грузов от поставщика к потребителям, что широко используется в энергетике, возникают вопросы наиболее рациональной их организации. Часто требуется найти такой план перевозок, при котором стоимость перевозок была бы минимальной. Такая задача называется транспортной задачей (Т3) по критерию стоимости.

В общем виде транспортная задача формулируется так: имеется $\mathrm{m}$ поставщиков и $\mathrm{n}$ потребителей однородного груза. Запасы i-го поставщика обозначим $a_{i}$, спрос j-го потребителя $-b_{j}$. Если обозначить $C_{i j}$ - стоимость перевозки единицы груза, a $x_{i j}-$ количество перевозимого груза от i-го поставщика j-му потребителю, то математическая модель задачи будет иметь следующий вид: 
1) суммарные запасы на перевозку должны быть минимальные:

$$
\sum_{i=1}^{m} \sum_{j=1}^{n} c_{i j} \cdot x_{i j} \rightarrow \min ;
$$

2) объем поставок і-го поставщика равен его запасу:

$$
\sum_{j=1}^{n} x_{i j}=a_{i}, \quad i=\overline{1, m} ;
$$

3) объем поставок ј-му потребителю равен его спросу:

$$
\sum_{i=1}^{m} x_{i j}=b_{j}, \quad i=\overline{1, n} ;
$$

4) неотрицательность переменных:

$$
x_{i j} \geq 0, \quad i=\overline{1, m}, \quad j=\overline{1, n} .
$$

Вместо матрицы затрат сіj может задаваться матрица расстояний $\mathrm{d}_{\mathrm{ij}}$.

Если суммарный объем отправляемых грузов равен потребности в этих грузах, то ТЗ называется закрытой (сбалансированной): $\sum_{\mathrm{i}=1}^{\mathrm{m}} \mathrm{a}_{\mathrm{i}}=\sum_{\mathrm{j}=1}^{\mathrm{n}} \mathrm{b}_{\mathrm{j}}$; иначе - открытой.

Если имеет место открытая Т3, ее нужно свести к закрытой форме следующим образом:

1) если спрос превышает предложение, то вводят фиктивного поставщика с недостающим объемом спроса; тарифы $\mathrm{c}_{\mathrm{ij}}$, связывающие фиктивные пункты с реальными, равны штрафам за недопоставку продукции;

2) если спрос меньше предложения, то вводят фиктивного потребителя с недостающим объемом потребления; элементы матрицы сіj, связывающие фиктивные пункты с реальными, равны стоимости хранения единицы нераспределенного груза. 
Если указанные в п. 1, 2 затраты неизвестны, то соответствующие элементы $c_{i j}=0$.

Транспортная задача решается в два этапа. Сначала необходимо найти исходный опорный план, а затем производится последовательно его улучшение до получения оптимального плана. На первом этапе для распределения ресурсов можно использовать правило «северо-западного угла» (здесь не учитываются тарифы и план далек от оптимального) или правило «минимального элемента», при котором необходимо осуществлять максимальные поставки ресурсов в клетки с минимальными тарифами. На втором этапе можно применить распределительный метод или метод потенциалов.

\section{2 Метод потенциалов}

Каждому поставщику (ограничению по запасам) поставим в соответствие потенциал $u_{i}(i=\overline{1, m})$, а каждому потребителю (ограничению по спросу) - потенциал $v_{j}(j=\overline{1, n})$.

Согласно теореме о потенциалах, каждой занятой клетке будет соответствовать уравнение $u_{i}+v_{j}=c_{i j}$. Так как всех занятых клеток должно быть $m+n-1$, т. е. на единицу меньше числа потенциалов, то для определения чисел $u_{i}, v_{j}$ необходимо решить систему из $m+n-1$ уравнений с $m+n$ неизвестными: $u_{i}+v_{j}=c_{i j}$. Одному из потенциалов задают обычно значение, равное нулю.

Для исследования плана на оптимальность по каждой свободной клетке проверяется условие $u_{i}+v_{j} \leq c_{i j}$. Если хотя бы одна свободная клетка не удовлетворяет данному условию, то опорный план не является оптимальным, его можно улучшить за счет загрузки этой клетки. Если таких клеток несколько, то наиболее перспективной для загрузки является клетка, для которой разность (оценка) между тарифом клетки и суммой потенциалов наименьшая, т. е. $\mathrm{S}_{\mathrm{ij}}=c_{\mathrm{ij}}-\left(\mathrm{u}_{\mathrm{i}}+\mathrm{v}_{\mathrm{j}}\right)<0$. 
Например, для клеток $(i ; k)$ и $(i ; t)$ имеем оценки: $S_{i k}=-5$, $\mathrm{S}_{\mathrm{it}}=-10$. Здесь наиболее потенциальной является клетка $(\mathrm{i} ; \mathrm{t})$. Экономически оценка показывает, на сколько денежных единиц уменьшатся транспортные издержки от загрузки данной клетки единицей груза. Эффективность плана от загрузки потенциальной клетки грузом в $\lambda$ единиц составит $\Delta \mathrm{F}=\mathrm{S}_{\mathrm{ij}} \cdot \lambda$ денежных единиц. Если для всех свободных клеток оценки $S_{i j} \geq 0$, то опорный план перевозок является оптимальным.

Итак, если для опорного плана перевозок указанное условие оптимальности не выполняется, то за счет загрузки свободной клетки с отрицательной оценкой план перевозок улучшается. Для наиболее перспективной свободной клетки строится замкнутый цикл с вершинами в загруженных клетках. Вершинам этого цикла условно приписываются знаки: свободной клетке - плюс, следующей по часовой или против часовой стрелки занятой клетке - минус, следующей - снова плюс и т. д. Из поставок в клетках цикла с «отрицательными» вершинами выбирается наименьшее количество $\lambda$ груза, которое и перемещается по клеткам этого цикла: прибавляется к поставкам в положительных вершинах и вычитается из поставок в отрицательных вершинах, в результате чего баланс цикла не нарушится.

Сформулируем алгоритм решения ТЗ методом потенциалов:

1) построить опорный план по одному из правил;

2) вычислить потенциалы поставщиков и потребителей $u_{i}$ и $v_{j}$ $(i=\overline{1, m}) ;(j=\overline{1, n})$, решив систему уравнений вида $u_{i}+v_{j}=c_{i j} ;$

3) вычислить оценки $S_{i j}$ для всех свободных клеток по формуле $\mathrm{S}_{\mathrm{ij}}=c_{\mathrm{ij}}-\left(\mathrm{u}_{\mathrm{i}}+\mathrm{v}_{\mathrm{j}}\right)$. Если все $\mathrm{S}_{\mathrm{ij}} \geq 0$, то полученный план является оптимальным. При этом если все $\mathrm{S}_{\mathrm{ij}} \geq 0$, то полученный оптимальный план единственный. В случае, если хотя бы одна оценка $S_{i j}=0$, имеем бесчисленное множество оптимальных планов с одним и тем же значением целевой функции.

ПРИМЕР 2.1. В трех хранилищах $\mathrm{A}_{1}, \mathrm{~A}_{2}, \mathrm{~A}_{3}$ имеется соответственно 70, 90 и 50 т топлива. Требуется спланировать пе- 
ревозку топлива четырем потребителям $\mathrm{B}_{1}, \mathrm{~B}_{2}, \mathrm{~B}_{3}, \mathrm{~B}_{4}$, спрос которых равен соответственно 50, 70, 40 и 40 т, так, чтобы затраты на транспортировку были минимальны. Стоимость перевозки 1 т указана в таблице.

\begin{tabular}{|c|c|c|c|c|c|}
\hline \multirow{3}{*}{ Хранилища } & \multicolumn{4}{|c|}{ Потребители } & \multirow{2}{*}{ Запас } \\
\hline & $\mathrm{B}_{1}$ & $\mathrm{~B}_{2}$ & $\overline{\mathrm{B}_{3}}$ & $\mathrm{~B}_{4}$ & \\
\hline & \multicolumn{4}{|c|}{ Стоимость перевозки 1 т топлива, ден. ед } & \\
\hline$A_{1}$ & 5 & 2 & 3 & 6 & 70 \\
\hline$\overline{A_{2}}$ & 4 & 3 & 5 & 7 & 90 \\
\hline $\mathrm{A}_{3}$ & 2 & 4 & 1 & 5 & 50 \\
\hline $\begin{array}{r}\text { Потребность } \\
\text { в топливе, т }\end{array}$ & 50 & 70 & 40 & 40 & $210>200$ \\
\hline
\end{tabular}

РЕШЕНИЕ. Поскольку запасы топлива в хранилищах превышают спрос потребителей, задача является открытой, вводится фиктивный потребитель, спрос которого $b_{5}=210-200=10$ т. Все затраты для фиктивного потребителя $c_{i 5}=0(i=\overline{1,3})$. После введения фиктивного потребителя открытая модель задачи преобразовалась в закрытую, а распределительная таблица принимает следующий вид:

\begin{tabular}{|c|c|c|c|c|c|c|}
\hline \multirow{2}{*}{ Хранилища } & \multicolumn{5}{|c|}{ Потребители } & \multirow{2}{*}{ Запас } \\
\cline { 2 - 6 } & $\mathrm{B}_{1}$ & \multicolumn{1}{|c|}{$\mathrm{B}_{2}$} & $\mathrm{~B}_{3}$ & $\mathrm{~B}_{4}$ & $\mathrm{~B}_{5}$ & топлива, т \\
\cline { 2 - 6 } & Стоимость перевозки 1 т топлива, ден. ед. & \\
\hline $\mathrm{A}_{1}$ & 5 & 2 & 3 & 6 & 0 & 70 \\
\hline $\mathrm{A}_{2}$ & 4 & 3 & 5 & 7 & 0 & 90 \\
\hline $\mathrm{A}_{3}$ & 2 & 4 & 1 & 5 & 0 & 50 \\
\hline $\begin{array}{c}\text { Потребность } \\
\text { в топливе, т }\end{array}$ & 50 & 70 & 40 & 40 & 10 & 210 \\
\hline
\end{tabular}

Исходный опорный план получим, например, по правилу «минимального элемента». Так как наименьшими являются нулевые тарифы для клеток $(1 ; 5),(2 ; 5),(3 ; 5)$, то загрузим первой, 
например, клетку $(1 ; 5), x_{15}=10$ т. Второй загружаем клетку (3; 3), $x_{33}=40$ т. Далее загружаем клетки $(1 ; 2),(3 ; 1),(2 ; 2),(2 ; 4)$, полагая $x_{12}=60 \mathrm{~T}, x_{31}=10 \mathrm{t}, x_{22}=10 \mathrm{~T}, x_{21}=40 \mathrm{~T}, x_{24}=10 \mathrm{~T}$.

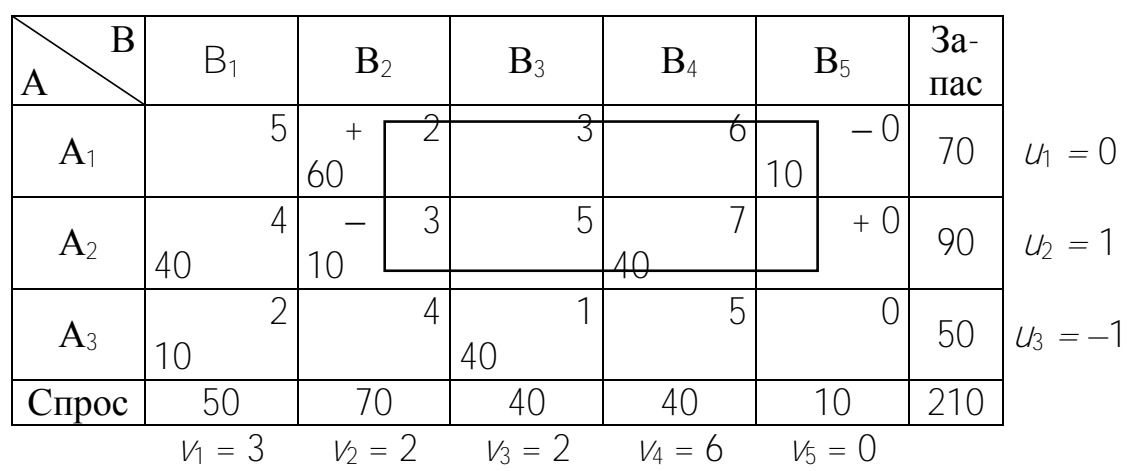

В результате распределения топлива по потребителям получили невырожденный план: условие для занятых клеток $\mathrm{m}+\mathrm{n}-1=3+5-1=7$ выполняется.

Для определения потенциалов составляем уравнения для занятых клеток: $u_{1}+v_{2}=2, u_{1}+v_{5}=0, u_{2}+v_{1}=4, u_{2}+v_{2}=3$, $u_{2}+v_{4}=7, u_{3}+v_{1}=2, u_{3}+v_{3}=1$. Положим, например, $u_{1}=0$, тогда $u_{2}=1, u_{3}=-1, v_{1}=3, v_{2}=2, v_{3}=2, v_{4}=6, v_{5}=0$.

Определим оценки свободных клеток:

$$
\begin{array}{ll}
S_{11}=5-(0+3)=2>0, & S_{25}=0-(0+2)=-2<0, \\
S_{13}=3-(0+2)=3>0, & S_{32}=4-(-1+2)=3>0, \\
S_{14}=6-(0+6)=0, & S_{34}=5-(-1+6)=0, \\
S_{23}=5-(1+2)=2>0, & S_{35}=0-(-1-0)=2>0 .
\end{array}
$$

Определив потенциалы, устанавливаем, что среди оценок свободных клеток одна отрицательная: $S_{25}=-1$, следовательно, план перевозок можно улучшить за счет загрузки клетки $(2 ; 5)$. Цикл для нее выделен линией в предыдущей таблице. 
Наименьшее количество топлива в отрицательных вершинах цикла равно 10 т. После смещения по циклу 10 т получаем новый план перевозок. Полученный план является вырожденным. Поставим число 0, например, в клетку $(2 ; 2)$.

\begin{tabular}{|c|c|c|c|c|c|c|c|}
\hline $\mathrm{B}$ & $\mathrm{B}_{1}$ & $\mathrm{~B}_{2}$ & $\mathrm{~B}_{3}$ & $\mathrm{~B}_{4}$ & $\mathrm{~B}_{5}$ & $\begin{array}{l}\text { 3a- } \\
\text { пас }\end{array}$ & \\
\hline $\mathrm{A}_{1}$ & 5 & $\begin{array}{ll}70 & 2 \\
\end{array}$ & 3 & 6 & 0 & 70 & $u_{1}=-1$ \\
\hline $\mathrm{A}_{2}$ & $\begin{array}{ll} & 4 \\
40 & \end{array}$ & 3 & 5 & $40 \quad 7$ & 10 & 90 & $\mathrm{u}_{2}=0$ \\
\hline $\mathrm{A}_{3}$ & $\begin{array}{ll} & 2 \\
10 & \end{array}$ & 4 & 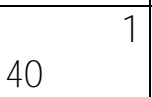 & 5 & 0 & 50 & $u_{3}=-2$ \\
\hline Спрос & 50 & 70 & 40 & 40 & 10 & 210 & \\
\hline
\end{tabular}

Для нового плана определяем новые потенциалы и находим оценки свободных клеток: $S_{11}=2>0, S_{13}=1>0, S_{14}=0, S_{15}=$ $=1>0, S_{23}=2>0, S_{32}=3>0, S_{34}=0, S_{35}=2>0$.

Оценки всех свободных клеток $\mathrm{S}_{\mathrm{ij}}>0$, следовательно, получен оптимальный план. Поскольку среди оценок имеются равные нулю, то за счет загрузки клеток $(1 ; 4),(3 ; 4)$ можно получить новые планы, но значение целевой функции не изменится. Это случай бесчисленного множества оптимальных планов.

Итак, в предыдущей таблице получили оптимальный план $X^{*}=\left[\begin{array}{cccc}0 & 70 & 0 & 0 \\ 40 & 0 & 0 & 40 \\ 10 & 0 & 40 & 0\end{array}\right]$, для которого значение целевой функции равно

$$
F\left(X^{*}\right)=2 \cdot 70+4 \cdot 40+7 \cdot 40+2 \cdot 10+1 \cdot 40=640 .
$$

Десять тонн топлива, находящегося в хранилище А2, осталось нераспределенным. 


\section{3. Транспортные задачи в усложненной постановке}

Рассмотренная выше постановка ТЗ в экономике предприятия встречается редко. Для того, чтобы свести задачу к задаче транспортного типа, приходится учитывать ряд дополнительных ограничений:

1. Отдельные поставки от определенных поставщиков некоторым потребителям должны быть исключены из-за отсутствия необходимых условий хранения, чрезмерной загрузки транспортных коммуникаций и т. Д. Это достигается путем искусственного завышения тарифов в тех ячейках транспортной таблицы, перевозки через которые следует запретить.

2. На предприятии необходимо оценить суммарные затраты на производство и транспортировку продукции. С подобной задачей сталкиваются при планировании размещения производственных объектов. С этой точки зрения может оказаться экономически более выгодным поставлять сырье из более отдаленных регионов, но по меньшей его себестоимости. В таких задачах в качестве критерия оптимальности принимают суммарные затраты на транспортировку и производство продукции (в транспортной таблице к стоимости перевозки добавляется себестоимость изготовления продукции).

3. Ряд транспортных маршрутов, по которым необходимо доставить груз, имеет ограничения по пропускной способности (ограничение «не более чем»).

Например, если по маршруту $\mathrm{A}_{i} \mathrm{~B}_{j}$ можно доставить не более чем q единиц груза, столбец $\mathrm{B}_{j}$ разбивается на два столбца: Bj $^{\prime}$ и Bj' $^{\prime}$. В первом спрос равен q, во втором - $\left(b_{j}-q\right)$.

Несмотря на то, что транспортные затраты в обоих столбцах одинаковы и равны исходным, ячейка $\mathrm{A}_{\mathrm{i}} \mathrm{B}_{\mathrm{j}}$ " блокируется (в ней ставится завышенный тариф).

4. Поставки по определенным маршрутам обязательны и должны войти в план поставок независимо от того, выгодно это или нет (ограничение «не менее чем»). В этом случае уменьшают запас груза и спрос у соответствующих поставщиков и 24 
потребителей на величину обязательных поставок и решают задачу относительно тех поставок, которые необязательны. После чего задачу корректируют с учетом обязательных поставок.

5. Необходимо максимизировать целевую функцию в Т3. Для этого надо изменить знак в тарифах на противоположный.

\section{Задание 2}

Задание, соответствующее номеру варианта (по порядковому номеру в списке группы), приведено в таблицах 2.1, 22

Нефтеперерабатывающие заводы 31, 32, 33, 34 и 35 ежедневно производят бензин, который направляется в бензохранилища $Б_{1}, \mathrm{БX}_{2}, Б_{3}, \mathrm{DX}_{4}, \mathrm{БX}_{5}$ и БХ 6 . Объем производства бензина и вместимость бензохранилищ представлены в таблице 2.1. Все бензохранилища связаны с заводами трубопроводами, по которым и перекачивается бензин. Стоимость перекачки 1 тыс. л бензина с заводов в бензохранилища приведена в таблице 2.1. Себестоимость 1 тыс. л бензина на нефтеперерабатывающих заводах соответственно равна 7, 4, 5, 8, 8 у. е.

Таблица 2.1

\begin{tabular}{|c|c|c|c|c|c|c|c|}
\hline \multirow{2}{*}{$\begin{array}{c}\text { Нефтеперераба- } \\
\text { тывающие заводы }\end{array}$} & \multicolumn{6}{|c|}{ Бензохранилища } & \multirow{2}{*}{ Запас, } \\
\cline { 2 - 7 } & \multicolumn{4}{|c|}{ Стоимость л } \\
\cline { 2 - 7 } & БХ $_{1}$ & БХ $_{2}$ & БХ & БХ & БХ & БХ & \\
\hline $3_{1}$ & 10 & 15 & 12 & 6 & 8 & 5 & 1500 \\
\hline $3_{2}$ & 10 & 13 & 4 & 5 & 10 & 6 & 650 \\
\hline $3_{3}$ & 8 & 6 & 6 & 6 & 18 & 3 & 390 \\
\hline $3_{4}$ & 4 & 8 & 11 & 9 & 20 & 14 & 450 \\
\hline $3_{5}$ & 5 & 7 & 8 & 8 & 12 & 7 & 560 \\
\hline Спрос, тыс. л & 800 & 1020 & 500 & 780 & 650 & 480 & \\
\hline
\end{tabular}

При этом необходимо учитывать, что из-за ремонтных работ временно нет возможности перевозить бензин с некоторых заводов в бензохранилища. В таблице 2.2 это показано в столбце «Запрет поставки» в формате [№ завода $\times$ № бензо- 
хранилища]. Например, «2×3» обозначает, что нельзя перевозить бензин с завода № 2 в бензохранилище № 3.

\section{Таблица 2.2}

\begin{tabular}{|c|c|c|c|c|}
\hline $\begin{array}{c}\text { Номер } \\
\text { вари- } \\
\text { анта }\end{array}$ & $\begin{array}{c}\text { Номера нефте- } \\
\text { перерабатываю- } \\
\text { щих заводов }\end{array}$ & $\begin{array}{c}\text { Номера } \\
\text { бензохра- } \\
\text { нилищ }\end{array}$ & $\begin{array}{c}\text { Запрет } \\
\text { поставки, } \\
\text { тыс. л }\end{array}$ & $\begin{array}{c}\text { Условие по поставке, } \\
\text { тыс. л }\end{array}$ \\
\hline 1 & $1,2,3,4$ & $1,2,3$ & $1 \times 2$ & $2 \times 3$ не более 100 \\
\hline 2 & $1,2,3$ & $1,2,3,4$ & $1 \times 1$ & $2 \times 4$ не более 500 \\
\hline 3 & $1,3,4$ & $1,2,3,5$ & $1 \times 3$ & $3 \times 2$ не менее 80 \\
\hline 4 & $1,2,3,5$ & $1,3,4$ & $1 \times 4$ & $2 \times 3$ не менее 300 \\
\hline 5 & $1,2,4,5$ & $1,4,5$ & $1 \times 5$ & $2 \times 4$ не более 200 \\
\hline 6 & $1,4,5$ & $1,4,5,6$ & $1 \times 6$ & $4 \times 4$ не менее 400 \\
\hline 7 & $3,4,5$ & $2,3,4,5$ & $3 \times 2$ & $4 \times 4$ не более 100 \\
\hline 8 & $1,2,5$ & $3,4,5,6$ & $1 \times 4$ & $5 \times 6$ не менее 350 \\
\hline 9 & $2,3,4,5$ & 1,2 & $2 \times 1$ & $3 \times 2$ не менее 90 \\
\hline 10 & 1,2 & $3,4,5,6$ & $1 \times 4$ & $2 \times 6$ не менее 450 \\
\hline 11 & $2,4,5$ & $1,2,3,4$ & $5 \times 2$ & $4 \times 3$ не менее 200 \\
\hline 12 & $1,3,5$ & $2,5,6$ & $1 \times 2$ & $3 \times 5$ не более 300 \\
\hline 13 & $2,4,5$ & $3,4,5,6$ & $4 \times 5$ & $5 \times 6$ не менее 180 \\
\hline 14 & $3,4,5$ & $4,5,6$ & $4 \times 5$ & $5 \times 4$ не менее 370 \\
\hline 15 & $1,2,3$ & $3,4,5$ & $3 \times 4$ & $2 \times 3$ не более 200 \\
\hline 16 & $1,3,4$ & $1,2,3,4$ & $4 \times 3$ & $4 \times 1$ не менее 100 \\
\hline 17 & $1,2,4,5$ & $4,5,6$ & $1 \times 5$ & $2 \times 5$ не менее 250 \\
\hline 18 & $3,4,5$ & $2,5,6$ & $5 \times 2$ & $3 \times 5$ не более 100 \\
\hline 19 & 1,5 & $1,3,5,6$ & $3 \times 3$ & $5 \times 5$ не менее 350 \\
\hline 20 & $1,3,5$ & $4,5,6$ & $1 \times 5$ & $5 \times 4$ не менее 90 \\
\hline 21 & $2,4,5$ & $1,3,5$ & $2 \times 3$ & $4 \times 3$ не менее 150 \\
\hline 22 & $3,4,5$ & $2,4,6$ & $3 \times 4$ & $4 \times 4$ не менее 400 \\
\hline 23 & $1,2,3$ & $1,2,3,4$ & $1 \times 2$ & $2 \times 3$ не более 100 \\
\hline 24 & $2,3,4$ & $4,5,6$ & $3 \times 5$ & $2 \times 6$ не менее 280 \\
\hline 25 & 1,3 & $1,2,3,4$ & $1 \times 2$ & $3 \times 3$ не более 170 \\
\hline 26 & $3,4,5$ & $2,5,6$ & $4 \times 2$ & $3 \times 5$ не более 200 \\
\hline 27 & $1,2,3,4$ & $4,5,6$ & $1 \times 6$ & $2 \times 5$ не менее 100 \\
\hline 28 & $1,3,4,5$ & $1,2,3$ & $1 \times 2$ & $5 \times 3$ не более 370 \\
\hline 29 & $3,4,5$ & $1,3,6$ & $3 \times 2$ & $5 \times 5$ не более 300 \\
\hline 30 & 2,4 & $3,4,5,6$ & $2 \times 3$ & $4 \times 6$ не менее 350 \\
\hline & & & & \\
\hline
\end{tabular}


Кроме того, необходимо учесть, что некоторые бензохранилища имеют договоры на гарантированную поставку бензина с определенных заводов в определенных объемах (условия «не более» или «не менее»). В таблице 2.2 это показано в столбце «Условие по поставке» в формате [№ завода $\times$ № бензохранилища «не более» / «не менее» объем поставки]. Например, «1×4 не менее 40» обозначает, что между заводом № 1 и бензохранилищем № 4 заключен договор на поставку не менее 40 тыс. л бензина.

Требуется составить план перекачки бензина с заводов в бензохранилища, обеспечивающий минимальные затраты.

Необходимо:

1) составить транспортную таблицу, позволяющую найти план перевозки бензина с заводов 3 в в бензохранилища БХ, отразив в ней все дополнительные условия;

2) найти исходный опорный план перевозки бензина;

3) решить задачу методом потенциалов, сформулировать вывод относительно распределения поставок бензина;

4) вычислить величину $F$ min минимальных суммарных затрат на производство и доставку бензина;

5) назвать пункты, в которых остается нераспределенная продукция, и указать объемы такой продукции.

\section{3. МЕТОДЫ СЕТЕВОГО ПЛАНИРОВАНИЯ И УПРАВЛЕНИЯ (МСПиУ)}

\section{1 Построение сети проекта}

При планировании сложных комплексов взаимосвязанных работ актуально использование МСПиУ.

Основой МСПиУ является сетевой график (сетевая модель), которая отражает логическую взаимосвязь и логическую взаимообусловленность всех входящих в проект элементарных операций (работ). 
Различают три вида событий: исходное - соответствует началу выполнения проекта, не имеет предшествующих работ; завериающее - соответствует достижению конечной цели, не имеет последующих работ; промежуточное - все остальные события.

Пока не будут завершены все входящие в событие работы, не может свершиться само событие и, следовательно, не может быть начата ни одна из выходящих из этого события работа. Событие - это момент времени, когда завершаются одни работы и начинаются другие. Событие представляет собой результат проведенных работ и, в отличие от работ, не имеет протяженности во времени.

Существует три вида работ (операций):

1) — действительная: работа, которая требует затрат времени и ресурсов, например, разработка проекта, выполнение СМР и т. д.;

2) - · - - операция ожидания: процесс, требующий только затрат времени, например, затвердевание бетона, естественная сушка краски и т. д.;

3) - - - - фиктивная работа или логическая зависимость: отражает ресурсную или логическую зависимость при выполнении некоторых операций. Фиктивная работа имеет нулевую продолжительность.

Работа называется критической, если она должна начинаться и заканчиваться в строго отведенное время, т. е. не имеет резерва времени своего начала и окончания, который не влиял бы на продолжительность выполнения всего проекта.

Для некритических работ возможен некоторый сдвиг времени их начала, но в определенных пределах, которые не влияют на срок выполнения всего проекта.

При построении сетевого графика необходимо следовать правилам: длина стрелки не зависит от времени выполнения работы; стрелка может не быть прямолинейным отрезком; для действительных работ используются сплошные, а для фиктив- 
ных - пунктирные стрелки; каждая операция должна быть представлена только одной стрелкой; между одними и теми же событиями не долюнно быть параллельных работ, т. е. работ с одинаковыми кодами; следует избегать пересечения стрелок; не должно быть стрелок, направленных справа налево; номер начального события должен быть меньше номера конечного события; не должно быть висячих событий (т. е. не имеющих предшествующих событий), кроме исходного; не должно быть mупиковых событий (т. е. не имеющих последующих событий), кроме завершающего; не должно быть циклов.

\section{2 Анализ проектов методом критического пути. \\ Расчет временных параметров сетевого графика. График Ганта}

Введем следующие обозначения:

$t_{p}(j)$ - самое раннее возможное время свершения ј-го события;

$t_{\Pi}(j)$ - самое позднее возможное время свершения j-го события;

$\mathrm{d}_{\mathrm{ij}}$ - длительность работы (i, j).

Расчет временных параметров сетевого графика проходит в два этапа:

1. Вычисляются ранние сроки свершения событий.

$t_{p}(1)=0$. Для узла ј определим узлы $p, q, \ldots, v$, которые связаны с узлом ј работами $(p, j),(q, j), \ldots,(v, j)$ и для которых уже вычислены самые ранние сроки свершения начальных событий, тогда

$$
t_{p}(j)=\max \left\{t_{p}(p)+d_{p j} ; t_{p}(q)+d_{q j} ; \ldots ; t_{p}(v)+d_{v j}\right\} .
$$

Первый этап заканчивается, когда будет вычислен tр послед-

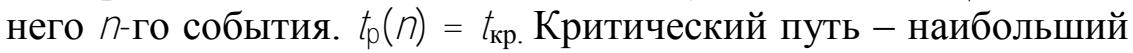
путь от начального события до завершающего. 
2. Вычисляются поздние сроки свершения событий.

Полагаем, что $t_{n}(n)=t_{p}(n)=t_{к р}$. Для узла $j$ определим узлы $p, q, \ldots, v$, которые связаны с узлом ј работами $(j, p),(j, q), \ldots$, $(j, v)$ и для которых уже вычислены самые поздние сроки свершения соответствующих событий

$$
t_{\Pi}(j)=\min \left\{t_{\Pi}(p)-d_{j p} ; t_{\Pi}(q)-d_{j q} ; \ldots ; t_{\Pi}(v)+d_{j v}\right\} .
$$

Второй этап заканчивается, когда будет вычислено $t_{п}(1)=0$. Резервы времени событий:

$$
R(j)=t_{\Pi}(j)-t_{p}(j) .
$$

Рассчитанные численные значения временных параметров записываются прямо в вершины сетевого графика (рисунок 3.1).

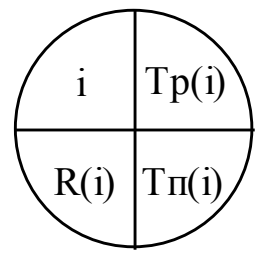

Рисунок 3.1 - Отображение временных параметров событий в вершинах сетевого графика

Для критических работ должна получиться непрерывная последовательность от начального события до завершающего. Сумма продолжительностей работ, лежащих на критическом пути, равна минимальному сроку выполнения проекта и равна $t_{\text {кр. }}$.

Резервы времени событий, лежащих на критическом пути, равны нулю. Для сетевого графика может быть несколько критических путей.

Удобным дополнением к сетевому графику является линейный график (график Ганта). На таком графике каждая работа изображается горизонтальным отрезком в привязке к оси времени, длина которого равна продолжительности выполне- 
ния работы. Начало каждой работы совпадает с ранним сроком свершения ее начального события. Критические работы образуют на графике Ганта непрерывный путь от начала выполнения проекта до его завершения без временных зазоров и перекрытий. Их суммарная длительность равна длительности выполнения всего проекта.

Некритические работы предпочитают начинать в самый ранний возможный срок, в этом случае остается запас времени, который можно использовать для решения неожиданно возникающих в ходе выполнения проекта проблем. Вместе с тем, можно перенести начало выполнения какого-либо некритического процесса.

\section{3. Оптимизация сетевых моделей по ресурсам (исполнителям)}

При оптимизации использования ресурса рабочей силы чаще всего сетевые работы стремятся организовать таким образом, чтобы количество одновременно занятых исполнителей было минимальным; выровнять потребность в людских ресурсах на протяжении срока выполнения проекта.

Суть оптимизации загрузки сетевых моделей по ресурсам заключается в следующем: необходимо таким образом организовать выполнение сетевых работ, чтобы количество одновременно работающих исполнителей было минимальным. Для проведения подобных видов оптимизации необходимо построить и проанализировать график привязки (график Ганта) и график загрузки.

График Ганта отображает взаимосвязь выполняемых работ во времени и строится на основе данных о продолжительности работ. По вертикальной оси графика привязки откладываются коды работ, по горизонтальной оси - длительность работ.

На графике загрузки по горизонтальной оси откладывается время, например в днях, по вертикальной - количество человек (ресурсов), занятых работой в каждый конкретный день. 
Описанные виды оптимизации загрузки выполняются за счет сдвига во времени некритических работ, т. е. работ, имеющих полный и/или свободный резервы времени. Полный и свободный резервы любой работы можно определить без специальных расчетов, анализируя только график привязки. Сдвиг работы означает, что она будет выполняться уже в другие дни (т. е. изменится время ее начала и окончания), что в свою очередь приведет к изменению количества исполнителей, работающих одновременно (т. е. уровня ежедневной загрузки сети).

ПРИМЕР 3.1. Для выполнения комплекса операций по ремонту энергетического оборудования предприятие в первые три дня выделяет 7 единиц ресурсов (ед. рес.), в 4 и 5 дни 6 ед. рес., в последующие -8 ед. рес. Сетевой график представлен на рисунке 3.2. Каждой работе графика приписаны два числа: 1) временная оценка, дней; 2) интенсивность потребления ресурса, ед. рес. Работа $(1,2)-3 ; 4 ;(1,3)-5 ; 5 ;(1,4)-7 ; 2$; $(2,3)-2 ; 3 ;(2,4)-4 ; 4 ;(3,4)-4 ; 1$. Определить сроки выполнения операций таким образом, чтобы завершить весь комплекс работ за минимальное время, при условии, что операции не допускают перерывов в выполнении.

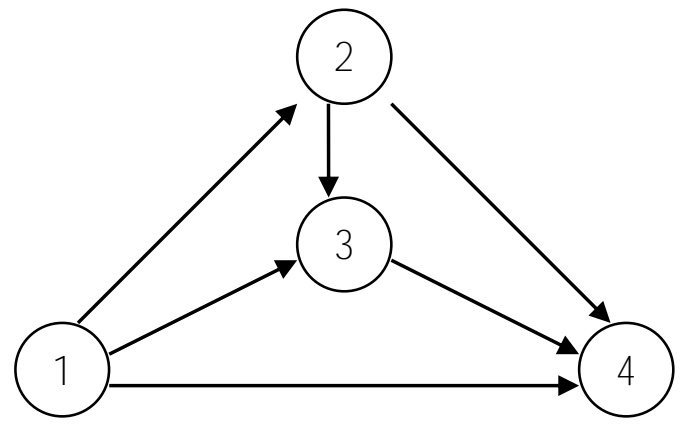

Рисунок 3.2 - Сетевой график выполнения комплекса работ

\section{РЕШЕНИЕ.}

1. Рассчитав временные параметры сетевого графика, определили, что весь проект может быть выполнен за $t_{\text {кр }}=9$ дней. 
На критическом пути лежат работы $(1,2),(2,3),(3,4)$. Представим график Ганта и график загрузки на рисунке $3.3, a$. Из графика загрузки видно, что в первые пять дней потребность в ресурсах больше их наличия (на графике выделено серым цветом). Следовательно, выполнить проект за девять дней невозможно, поэтому необходимо провести оптимизацию по ресурсам, чтобы выполнить работы с помощью имеющихся ресурсов.

2. Проецируем на ось времени начало и окончание каждой работы. Проекцию, совпадающую с началом координат, обозначим $t_{0}=0, t_{1}=3-$ окончание работы $(1,2)$. Определим полные резервы времени $\mathrm{R}_{\mathrm{ij}}$ операций, расположенных на проме-жутке от $t_{0}$ до $t_{1}$, нумеруем эти операции в порядке возрастания полных резервов. Операции с одинаковыми резервами времени нумеруют в порядке убывания интенсивности. $\mathrm{R}_{12}=$ $0, R^{\Pi} 13=0, R^{\Pi_{14}}=9-7=2$ дня. Нумеруем работы по важности: I - $(1,3), I I-(1,2), I I I-(1,4)$.

3. Последовательно суммируем интенсивности работ, расположенных над промежутком от $t_{0}$ до $t_{1}$ в порядке возрастания их номеров и сравниваем полученные суммы с заданной величиной имеющихся ресурсов R. Все операции, сумма интенсивностей которых не превышает наличие ресурсов $R$, оставляем в первоначальном положении. Если после добавления интенсивности какой-либо операции окажется, что суммарное потребление ресурсов больше $\mathrm{R}$, то эту операцию сдвигают вправо на величину рассматриваемого промежутка. Переходят к добавлению интенсивности следующей операции, расположенной на промежутке от to до $t_{1}$. Результатом выполнения этого действия будет новый график Ганта, момент $\mathrm{t}_{1}$ которого считаем началом оставшейся части комплекса операций (рисунок 3.3, б). Операции (i, j), расположенные над промежутком от to $_{0}$ до $t_{\text {кр, }}$ изображают так, чтобы их начала совпадали с новыми сроками свершения событий. 

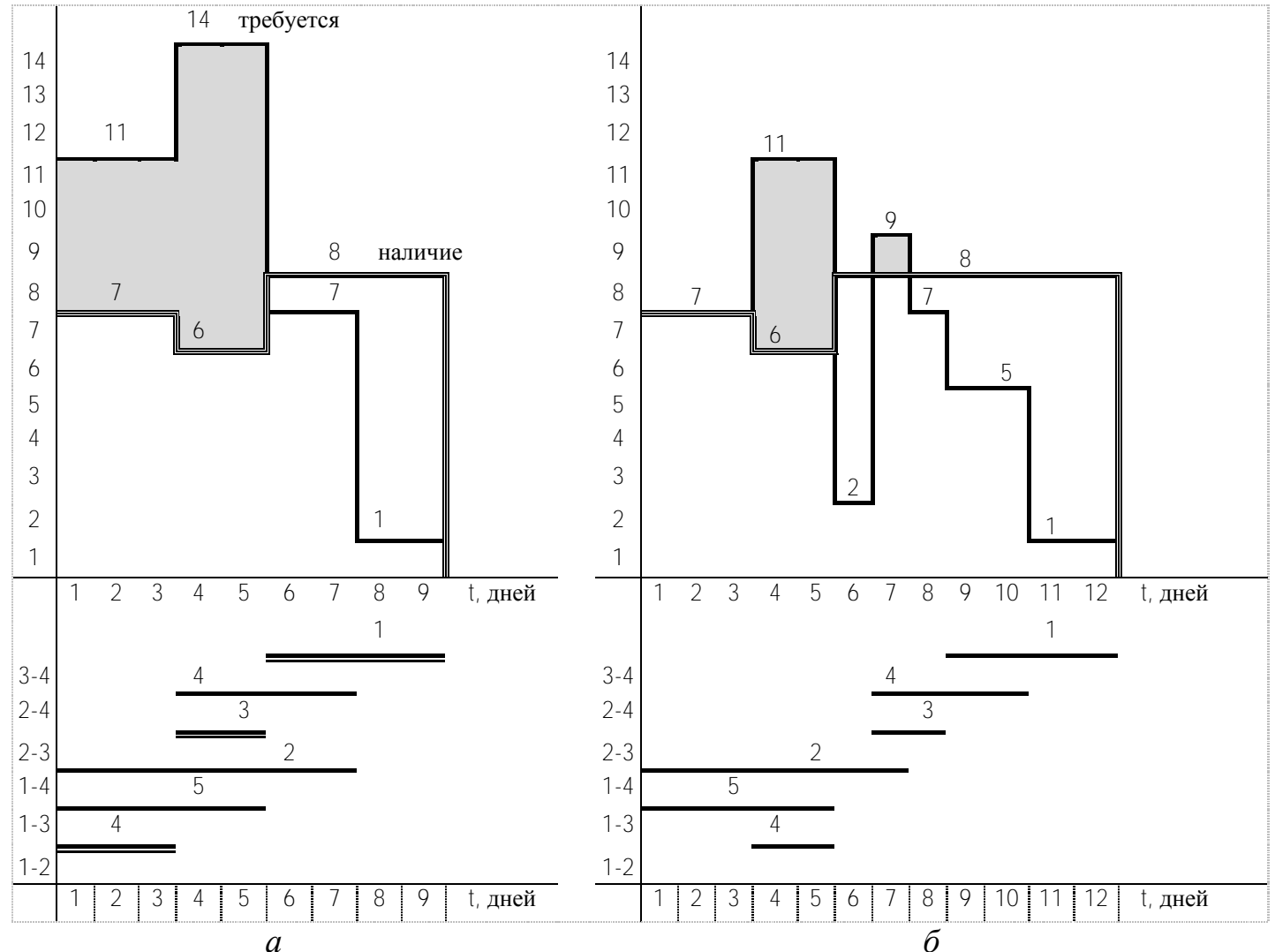

Рисунок 3.3 - График Ганта $(a)$ и график загрузки (б) 
4. Проецируем на ось времени начало и окончание операций,

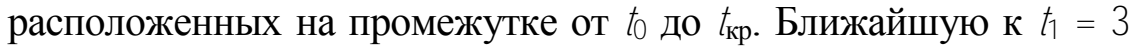
проекцию обозначим $t_{2}=5$ дней. Определим полные резервы операций, расположенных на промежутке от $\mathrm{t}_{1}$ до $_{2}$ и нумеруем их. Сначала нумеруют операции, начатые левее момента $t_{1}$ согласно возрастанию разностей между полными резервами времени этих операций и длительностью от начала до $t_{2}$. Операции с одинаковыми разностями нумеруют в порядке убывания интенсивностей. Все остальные операции нумеруют как в п. 2. Выполняют действия, аналогичные действиям из п. 3. Если сдвигается операция, начатая левее $t_{1}$, начало ее устанавливают в $t_{2}$.

$R^{{ }^{n}}{ }_{13}-L_{13}=3-5=2, R^{{ }^{n}} 14-L_{14}=5-5=0, R^{{ }_{1}} 12=0$. Нумеруем работы по важности: I - $(1,3), I I-(1,4), I I I-(1,2)$. Отмечаем работы на новом графике Ганта согласно данной нумерации (рисунок $3.4, a$ ).

Далее аналогично рассматриваем промежутки от $\mathrm{t}_{2}=5$ до $\mathrm{t}_{3}=8$, от $\mathrm{t}_{3}=8$ до $\mathrm{t}_{4}=10$, от $\mathrm{t}_{4}=10$ до $\mathrm{t}_{5}=14$ и выполняем действия пп. 2-4 (рисунок $3.4,6$ ). После каждого графика Ганта необходимо провести проверку графика загрузки, чтобы определить, достаточно ли имеющихся ресурсов для выполнения комплекса работ.

\section{Задание 3}

Задание, соответствующее номеру варианта (по порядковому номеру в сииске группы), приведено в таблицах 3.1, 3.2.

Дана последовательность работ по разработке стенда.

Необходимо:

1) на основании данных таблицы 3.1 составить сетевой график комплекса работ;

2) рассчитать временные параметры графика работ, определить критический путь, назвать работы, лежащие на критическом пути;

3) построить график Ганта и график загрузки;

4) провести оптимизацию комплекса работ по ресурсам (исполнителям). 


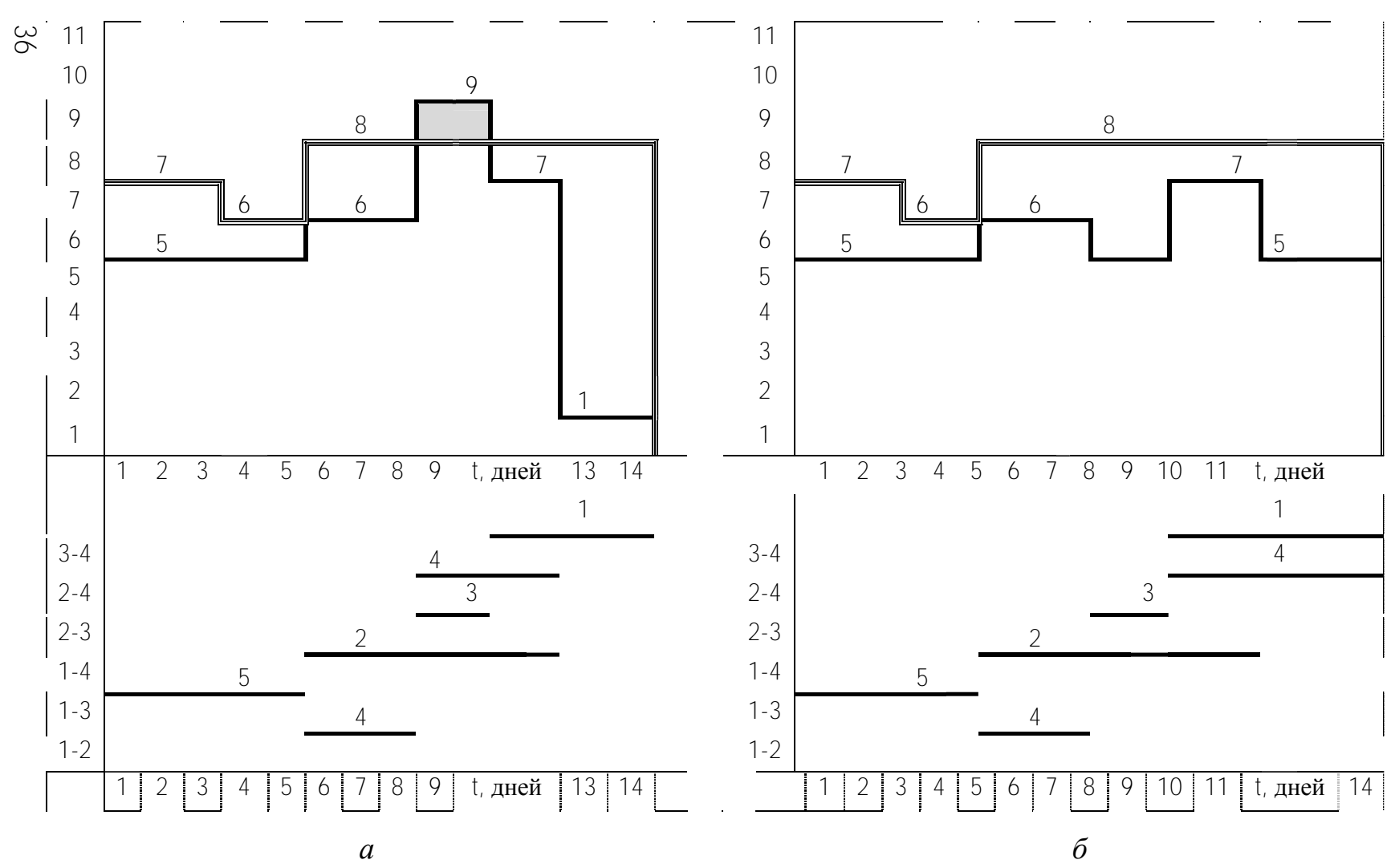

Рисунок 3.4 - График Ганта (a) и график загрузки (б) 


\section{Таблица 3.1}

\begin{tabular}{|c|c|c|c|c|c|c|c|}
\hline 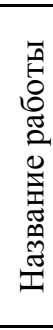 & Содержание работы & 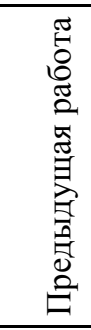 & 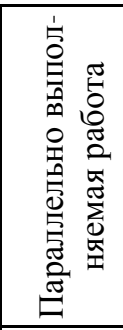 & 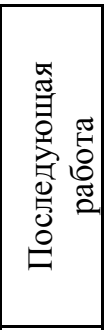 & 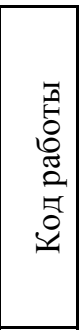 & 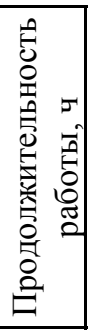 & 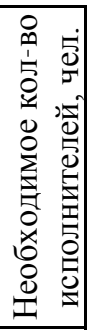 \\
\hline A & $\begin{array}{l}\text { Разработка технических } \\
\text { условий на стенд }\end{array}$ & - & - & $B, C$ & 1,2 & 2 & 3 \\
\hline $\mathrm{B}$ & Общая компоновка стенда & $A$ & $\mathrm{C}$ & $D, E, F$ & 2,3 & 2 & 3 \\
\hline C & $\begin{array}{l}\text { Разработка и выдача ТЗ на со- } \\
\text { ставление рабочей докумен- } \\
\text { тации по эксплуатации стенда }\end{array}$ & A & $\begin{array}{l}\mathrm{B}, \mathrm{D}, \mathrm{E} \\
\mathrm{F}, \mathrm{G}, \mathrm{H}, \mathrm{I}\end{array}$ & $L$ & 2,8 & 3 & 3 \\
\hline D & $\begin{array}{l}\text { Разработка технологии изго- } \\
\text { товления электрической } \\
\text { части стенда }\end{array}$ & B & $E, F$ & G & 3,4 & 5 & 5 \\
\hline $\mathrm{E}$ & $\begin{array}{l}\text { Разработка технологии изго- } \\
\text { товления механической части } \\
\text { стенда }\end{array}$ & B & $D, F$ & $\mathrm{H}$ & 3,5 & 4 & 4 \\
\hline $\mathrm{F}$ & $\begin{array}{l}\text { Оформление и размещение } \\
\text { заказов на покупные эле- } \\
\text { менты, необходимые для } \\
\text { сборки стенда }\end{array}$ & B & $D, E$ & I & 3,6 & 3 & 2 \\
\hline G & $\begin{array}{l}\text { Изготовление электрической } \\
\text { части стенда }\end{array}$ & D & $\mathrm{H}, \mathrm{I}$ & $\mathrm{J}, \mathrm{K}$ & 4,7 & 10 & 6 \\
\hline $\mathrm{H}$ & $\begin{array}{l}\text { Изготовление механической } \\
\text { части стенда }\end{array}$ & $E$ & $G, I$ & $\mathrm{~J}, \mathrm{~K}$ & 5,7 & 12 & 8 \\
\hline I & $\begin{array}{l}\text { Выполнение заказов на по- } \\
\text { купные элементы }\end{array}$ & $\mathrm{F}$ & $H, G$ & $\mathrm{~J}, \mathrm{~K}$ & 6,7 & 6 & 1 \\
\hline J & $\begin{array}{l}\text { Передача информации о ха- } \\
\text { рактеристиках стенда для раз- } \\
\text { работки рабочей документа- } \\
\text { ции по эксплуатации стенда }\end{array}$ & $\mathrm{G}, \mathrm{H}, \mathrm{I}$ & K & $\mathrm{L}$ & 7,8 & 0 & 1 \\
\hline K & Сборка стенда & $\mathrm{G}, \mathrm{H}, \mathrm{I}$ & $\mathrm{J}, \mathrm{L}$ & M & 7,9 & 7 & 4 \\
\hline $\mathrm{L}$ & $\begin{array}{l}\text { Разработка рабочей докумен- } \\
\text { тации по эксплуатации стенда }\end{array}$ & $\mathrm{C}, \mathrm{J}$ & K & M & 8,9 & 6 & 5 \\
\hline M & $\begin{array}{l}\text { Контрольные испытания } \\
\text { стенда }\end{array}$ & $L, K$ & - & - & 9,10 & 3 & 3 \\
\hline
\end{tabular}




\section{Таблица 3.2}

\begin{tabular}{|c|c|c|c|c|c|}
\hline \multirow{2}{*}{$\begin{array}{c}\text { Номер } \\
\text { вари- } \\
\text { анта }\end{array}$} & \multicolumn{3}{|c|}{$\begin{array}{l}\text { Имеющееся количество } \\
\text { исполнителей (человек) }\end{array}$} & \multirow{2}{*}{$\begin{array}{l}\text { Работы допус- } \\
\text { кают перерыв } \\
\text { в выполнении }\end{array}$} & \multirow{2}{*}{$\begin{array}{c}\text { Работы не допус- } \\
\text { кают перерыв } \\
\text { в выполнении }\end{array}$} \\
\hline & 1-4 дни & 5-20 дни & 21-30 дни & & \\
\hline 1 & 5 & 15 & 12 & + & - \\
\hline 2 & 4 & 20 & 8 & + & - \\
\hline 3 & 5 & 10 & 20 & + & - \\
\hline 4 & 7 & 10 & 20 & + & - \\
\hline 5 & 6 & 14 & 7 & + & - \\
\hline 6 & 4 & 16 & 9 & - & + \\
\hline 7 & 4 & 16 & 16 & - & + \\
\hline 8 & 6 & 13 & 16 & - & + \\
\hline 9 & 5 & 17 & 10 & - & + \\
\hline 10 & 8 & 14 & 19 & - & + \\
\hline 11 & 9 & 13 & 10 & + & - \\
\hline 12 & 4 & 11 & 20 & - & + \\
\hline 13 & 7 & 9 & 5 & + & - \\
\hline 14 & 6 & 8 & 17 & - & + \\
\hline 15 & 5 & 14 & 15 & + & - \\
\hline 16 & 4 & 17 & 8 & - & + \\
\hline 17 & 6 & 13 & 9 & + & - \\
\hline 18 & 3 & 15 & 8 & - & + \\
\hline 19 & 6 & 10 & 20 & + & - \\
\hline 20 & 12 & 12 & 12 & + & - \\
\hline 21 & 10 & 13 & 10 & + & - \\
\hline 22 & 4 & 17 & 12 & - & + \\
\hline 23 & 8 & 10 & 16 & - & + \\
\hline 24 & 6 & 19 & 19 & - & + \\
\hline 25 & 7 & 14 & 14 & + & - \\
\hline 26 & 5 & 17 & 12 & - & + \\
\hline 27 & 3 & 17 & 17 & + & - \\
\hline 28 & 4 & 19 & 15 & - & + \\
\hline 29 & 9 & 13 & 13 & + & - \\
\hline 30 & 8 & 10 & 15 & - & + \\
\hline
\end{tabular}




\section{ЛИТЕРАТУРА}

1. Балашевич, В.А. Экономико-математическое моделирование производственных систем: учебное пособие для вузов / В.А. Балашевич, А.М. Андронов. - Минск: Універсітэцкае, 1995. - 240 с.: ил.

2. Кузнецов, А.В. Высшая математика. Математическое программирование: учебник / А.В. Кузнецов, В.А. Сакович, Н.И. Холод. - 2-е изд., перераб. и доп. - Минск: Выш. школа, 2001. 351 с.: ил.

3. Падалко, Л.П. Математические методы оптимального планирования развития и эксплуатации энергосистем / Л.П. Падалко. - Минск: Выш. школа, 1972.

4. Партыка, Т.Л. Математические методы: учебник / Т.Л. Партыка, Попов И.И. - М.: ФОРУМ: ИНФРА-М, 2005. - 464 с.: ил.

5. Экономико-математические методы и модели: учебное пособие / Н.И. Холод [и др.]; под ред. А.В. Кузнецова. - 2-е изд. Минск: БГЭУ, 2000. - 412 с.: ил. 


\section{СОДЕРЖАНИЕ}

\section{1. ДЕТЕРМИНИРОВАННЫЕ ЛИНЕЙНЫЕ}

МОДЕЛИ. . . . . . . . . . . . . . . . . . . 3

1.1. Постановка задачи линейного программирования. . .. . . . . . . . . . . . . . 3

1.2. Анализ линейных моделей на чувствительность (устойчивость). . . . . . . . . . . . . . . 4

1.3. Экономическая интерпретация решения задач ЛП. . . . . . . . . . . . . . . . . . . . . . . . . . 6

1.4. Экономическая интерпретация двойственности. . . 10 Задание $1 . \ldots \ldots \ldots \ldots \ldots \ldots \ldots$. . . . . . . . . . . . . . . 14

2. ТРАНСПОРТНЫЕ МОДЕЛИ. . . . . . . . . . . . . . . . . . . 17

2.1. Транспортная задача и ее особенности. . . . . . . . . . 17

2.2. Метод потенциалов. . . . . . . . . . . . . . . . . . . . . . 19

2.3. Транспортные задачи в усложненной постановке. . . . . . . . . . . . . . . . . . . . . . . . . . 24

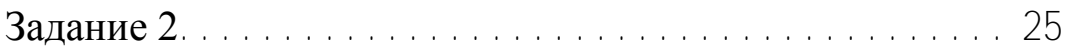

3. МЕТОДЫ СЕТЕВОГО ПЛАНИРОВАНИЯ И УПРАВЛЕНИЯ (МСПиУ), . . . . . . . . . . . . . . . . . 27

3.1. Построение сети проекта. . . . . . . . . . . . . . . . . 27

3.2. Анализ проектов методом критического пути.

Расчет временных параметров сетевого графика.

График Ганта. . . . . . . . . . . . . . . . . . . . . . 29

3.3. Оптимизация сетевых моделей по ресурсам (исполнителям). . . . . . . . . . . . . . . . . . . 31

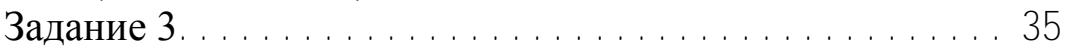
Литература. . . . . . . . . . . . . . . . . . . . . . . . . . 39 
Учебное издание

\section{ЭКОНОМИКО-МАТЕМАТИЧЕСКИЕ МЕТОДЫ И МОДЕЛИ}

Методические указания к контрольным работам для студентов заочной формы обучения специальности 1-270101 «Экономика и организация производства» направления 1-27 01 01-10 «Энергетика»

Составители:

НАГОРНОВ Виктор Николаевич КУПРИК Алёна Викторовна

Редактор Т.А. Подолякова

Компьютерная верстка Н.А. Школьниковой

Подписано в печать 10.05.2011.

Формат $60 \times 84^{1} / 16$. Бумага офсетная.

Отпечатано на ризографе. Гарнитура Таймс.

Усл. печ. л. 2,38. Уч.-изд. л. 1,86. Тираж 100. Заказ 832.

Издатель и полиграфическое исполнение:

Белорусский национальный технический университет.

ЛИ № 02330/0494349 от 16.03.2009.

Проспект Независимости, 65. 220013, Минск. 